\title{
Trois points de vue
}

Rui Canário, Agnès van Zanten et Alan Wagner

\section{(2) OpenEdition}

Journals

Édition électronique

URL : https://journals.openedition.org/ries/1356

DOI : 10.4000/ries.1356

ISSN : 2261-4265

Éditeur

France Education international

Édition imprimée

Date de publication : 1 décembre 2005

Pagination : 137-150

ISSN : 1254-4590

\section{Référence électronique}

Rui Canário, Agnès van Zanten et Alan Wagner, "Trois points de vue », Revue internationale d'éducation de Sèvres [En ligne], 40 | décembre 2005, mis en ligne le 17 novembre 2011, consulté le 08 juillet 2021. URL : http://journals.openedition.org/ries/1356 ; DOI : https://doi.org/10.4000/ries.1356 


\section{Trois points de vue}

\section{Rui Canario}

L'autonomie de l'établissement scolaire est-elle un facteur d'efficacité pédagogique et de réussite des élèves? Comment l'école s'adapte-t-elle à la diversité des élèves? La synthèse des débats des deux ateliers auxquels j'ai participé comme observateur n'est pas une tâche facile : les questions sont complexes et les contributions très riches et diversifiées. Mon exposé a été construit à partir d'une lecture préalable des textes des différents pays et, surtout, de l'écoute attentive des communications et des débats. Pourtant, il n'est pas possible de rendre d'une manière neutre et objective ce qui a été dit et discuté. Ma synthèse exprime, nécessairement, un point de vue personnel.

Depuis la Deuxième Guerre Mondiale, il y a eu, partout dans le monde, une croissance exponentielle des systèmes scolaires. Cela s'est traduit, d'une part, par une massification qui pose avec une acuité particulière le problème de la diversité. D'autre part, le changement d'échelle en matière de dimension des systèmes scolaires pose un autre problème, celui de la gouvernabilité qui est à mon avis au cœur de la problématique actuelle de l'autonomie des établissements. Les débats auxquels j'ai pu assister ont confirmé cette idée : les deux problèmes font système et la construction d'une solution ne peut pas les dissocier.

Une première grande conclusion qu'on peut retirer des débats dans les deux ateliers, c'est l'idée du rôle crucial de l'établissement scolaire comme unité stratégique de référence, comme point critique et comme niveau privilégié pour l'intervention et pour la prise de décision si l'on veut produire des changements. Derrière le débat et les différentes interventions dans les ateliers, on peut dresser un constat qui n'a pas été toujours explicite : c'est l'idée d'une érosion ou d'un épuisement progressif d'un modèle d'école envisagé comme un cadre d'accueil unique, dispensant à tout le monde un enseignement uniforme. Il faut accepter ce constat à mon avis incontournable, pour essayer de mettre en équation les deux problèmes : celui de la diversité et celui de l'autonomie.

\section{La question de la diversité des élèves}

Tout d'abord, il faut noter que le problème de la diversité existe depuis l'invention de l'école. Cette difficulté ou cette incapacité à affronter la diversité est une contradiction inhérente à l'École car elle a été construite, historiquement, contre la diversité linguistique, culturelle, et les différents particularismes. Aujourd'hui, et depuis plusieurs années nous le savons bien, la diversité des publics est croissante en raison de facteurs exogènes à l'école (comme les problèmes des migrations, les problèmes démographiques, les problèmes politiques par exemple), mais également en raison de facteurs endogènes à l'école, c'est-à-dire que l'école fabrique une 
population composée par différents groupes qui posent problème à son fonctionnement. Simultanément on peut dire, et je cite ici notre collègue de Belgique francophone, qu' « une culture anti-hétérogène continue à modeler au quotidien le terrain éducatif». Elle en tire la conclusion suivante : «le système est malade de son hétérogénéité». Je pense que cela s'applique à un grand nombre de pays.

La réponse qui est donnée la plupart du temps à ce problème «d'adaptation» de l'école à la diversité croissante et publique consiste à récréer de l'homogénéité. J'utilise ici, une nouvelle fois, une formule heureuse du texte de Belgique francophone : à l'école, «qui se ressemble, s'assemble». Par des biais différents comme les filières de rattrapage, le redoublement, les pédagogies de soutien, les mécanismes de quasi-marché (qui conduiraient à donner une «liberté» de choix au consommateur), on a mis en place une pluralité de solutions qui s'inspirent surtout d'une perspective compensatoire. Elles ont une visée orthopédique par rapport à des élèves ou à des groupes d'élèves qui sont vus, essentiellement, à partir d'une lecture négative qui met en évidence leurs lacunes. Ils sont placés dans une situation de déficit.

Cela se traduit, dans la plupart des situations, par des effets indésirables, c'est-à-dire "pervers». D'une part, au niveau social, on constate des phénomènes de ségrégation et d'exclusion qui se traduisent par des orientations massives vers des filières spéciales et, dans certains pays, par une désaffection claire pour l'école publique de la part surtout des classes moyennes. On observe, d'autre part, au niveau pédagogique, ce qu'on peut appeler une vision "pathologique» des difficultés scolaires vues comme quelque chose qui échappe à la règle, qui est déviant et qui exige, donc, des mesures exceptionnelles.

\section{La question de l'autonomie}

Deuxièmement, comment se pose le problème de l'autonomie? J'essaierai de montrer comment diversité et autonomie deviennent indissociables. Tout d'abord, une première contribution des débats dans l'atelier a été de reconnaître le besoin de complexifier la notion d'autonomie. On a retenu une distinction analytique importante, proposée par notre collègue portugais, sur l'autonomie «décrétée» et l'autonomie «construite», qui renvoient à des façons totalement différentes de penser et de lire les processus d'autonomie. D'autre part, on a également retenu la distinction entre plusieurs logiques d'autonomie : une logique administrative est, par exemple, très différente d'une logique politique ou d'une logique de marché.

En général, on a identifié des obstacles à la construction de l'autonomie, c'est-à-dire à l'autonomie construite à partir de l'initiative des acteurs locaux. Ces obstacles viennent de l'administration qui, partout, prend des mesures contradictoires. Sous la rhétorique de l'autonomie, des pratiques de centralisation sont en réalité introduites. Ces stratégies reviennent à exporter des problèmes vers la périphérie et à répondre ainsi au problème de la gouvernabilité des systèmes scolaires. Mais il y a également des obstacles très importants qui viennent de l'intérieur des établissements : on a signalé, notamment, la persistance d'une culture profession- 


\section{Trois points de vue}

nelle des enseignants liée à une certaine façon d'envisager leur métier et la division du travail qui en découle. Elle dépend étroitement d'une grammaire organisationnelle de l'établissement basée sur la classe et l'heure de cours, c'est-à-dire des éléments intériorisés depuis longtemps et conduisant à reproduire ces normes.

La pertinence de la liaison entre le problème de l'autonomie et le problème de la diversité découle du fait qu'on peut essayer de définir l'autonomie comme la capacité de chaque établissement à répondre à la diversité, en construisant des réponses éducatives sur mesure, c'est-à-dire, des réponses non standard, non uniformes, et pertinentes par rapport à des publics et à des contextes singuliers. Dans cette perspective, l'autonomie construite conduit à faire progressivement de la diversité une règle et non plus l'exception qui permet au système de répondre aux cas considérés comme déviants ou anormaux.

En général, l'autonomie tend, historiquement, à être pensée à partir du rapport entre l'établissement et l'administration centrale. Pourtant, le débat a mis en évidence une autre manière de poser le problème. Ce point n'a pas été très développé mais il me paraît très fécond et très important : il revient à penser l'autonomie comme le rapport de l'établissement avec son contexte. De ce point de vue, construire l'autonomie par rapport à l'administration équivaut à construire une dépendance étroite par rapport au contexte et aux publics. Cela n'est pas une nouveauté dans l'éducation car c'est la caractéristique même des expériences les plus innovantes dans le domaine de la formation des adultes par exemple, qui, par tradition, en se distanciant de la forme scolaire, devient nécessairement dépendante du public. On peut repenser le problème de la construction de l'autonomie dans cette perspective.

\section{Repenser la nature du travail scolaire}

Je terminerai en signalant un point qui me paraît, aussi, central. Pour tenter de penser la façon de construire, simultanément, l'autonomie et des stratégies de fonctionnement capables de répondre à la diversité, il faut toucher un point critique qui est la nature du travail scolaire. Tout se joue autour de la possibilité de penser un changement en profondeur du métier de l'élève. On doit penser à la question de la diversité et de l'autonomie par rapport à ce que l'élève fait dans l'établissement et à la façon dont le travail scolaire est organisé. Évidemment, cela aura des conséquences sur le métier d'enseignant et sur la grammaire de l'école, notamment la remise en cause d'une organisation basée sur la classe, l'heure de cours et d'un certain nombre de traces classiques de l'organisation scolaire.

Dans l'un et l'autre atelier, on a fait référence à des expériences et à des innovations de base. Cela nous autorise à poser une hypothèse qui est peut-être fertile, selon laquelle les innovations porteuses d'avenir sur la question de l'autonomie et de la diversité existent déjà quelque part à l'intérieur du système. Il faut les identifier, en tirer des enseignements et cela suppose une autre posture de la part de l'administration et des chercheurs. 


\section{Agnès van Zanten}

Comment les politiques éducatives s'adaptent-elles aux évolutions de la société? L'enseignant : acteur ou spectateur des évolutions? Les thèmes des deux ateliers étaient très proches : il s'agissait de voir comment il peut y avoir une autonomie du politique et une autonomie de l'enseignant dans un nouveau système de contraintes.

\section{Les politiques}

Avant d'en venir aux ateliers eux-mêmes, il y a une question préalable à laquelle je souhaiterais répondre.

\section{Les politiques s'adaptent-elles?}

La réponse du sociologue est sans ambiguïté : nous n'éduquons pas nos enfants comme nous le voulons, nous disait Émile Durkheim, et bien sûr les politiques ne peuvent pas faire ce qu'ils veulent. Il y a des forces sociales qui s'imposent à l'action des individus, qu'ils soient enseignants dans la classe, parents dans la famille ou politiques en train d'élaborer des orientations.

Cela étant, le rapport entre l'école et la société évolue selon les périodes et nous sommes dans une période très paradoxale parce que d'un côté, on voit de façon éclatante le triomphe de l'école, et de l'autre une école victime de son succès.

On peut observer le triomphe de toutes les politiques éducatives mises en œuvre depuis le XIX ${ }^{\mathrm{e}}$ siècle : l'élévation impressionnante des niveaux d'instruction; la connaissance qui est devenue un des piliers du développement culturel et économique; une forme de "pédagogisation» profonde des rapports sociaux qui fait que la forme scolaire est partout.

Mais en même temps que le modèle scolaire a triomphé, l'école est devenue victime de son succès. Les demandes sociales ne font que croître à son égard, elles proviennent d'acteurs qui sont beaucoup plus instruits et donc plus réflexifs et critiques à son égard.

Par ailleurs, il faut tenir compte de la diversité des contextes politiques. Un collègue italien nous rappelait comment, dans son pays, la faiblesse du système politique n'a pas permis une nouvelle réforme de l'éducation depuis celle de la scuola media il y a déjà plus de quarante ans. Les exemples de pays de l'Est comme la Hongrie ou la Roumanie montrent bien que les changements très brusques de régime, les alternances politiques successives ne permettent pas de mettre en place une politique cohérente. Pour les autres pays, même quand le système politique est stable, il existe toujours ce que les politologues appellent des «fenêtres d'opportunité». En conclusion, on ne peut pas apporter une réponse globale à la question posée. 


\section{Y a-t-il des changements dans les politiques éducatives?}

Nous avons bien sûr répondu «oui». De très nombreux exemples de changements ont été donnés dans les différents systèmes d'enseignement représentés dans l'atelier (Japon, Jordanie, Hongrie, Norvège, Québec et France). On constate partout une évolution des cadres normatifs de l'action, la transition d'une rhétorique de l'intégration et de la démocratisation vers des rhétoriques de la qualité, de l'efficacité, de la compétitivité et de l'individualisation des systèmes. Elles vont de pair avec des changements importants de structures : la décentralisation territoriale et pédagogique, l'autonomie des établissements, des modifications dans les programmes et les méthodes pédagogiques et des évolutions dans les modes d'élaboration des réformes.

\section{À quels changements sociaux ces transformations correspondent-elles?}

Parmi les thèmes évoqués, je rappelle juste la place de la nouvelle économie et la place du savoir dans cette nouvelle économie. Notre collègue japonais insistait sur le poids très fort de l'individualisme dans le système éducatif. Comme notre collègue français, il faisait un lien entre cet individualisme et la diffusion d'un modèle libéral.

Personnellement je serais plus nuancée. Le processus d'individualisation a commencé il y a fort longtemps et nous assistons actuellement à une nouvelle phase de son développement. Il faut remarquer la présence d'un individualisme instrumental avec des acteurs sociaux plus utilitaristes, plus stratèges, qui sont du côté du risque et du marché et qui, bien sûr, sont en phase très grande avec ce libéralisme économique; mais on ne peut ignorer aussi l'existence d'un individualisme expressif, comme au Japon par exemple, avec des acteurs de plus en plus demandeurs de reconnaissance, en particulier de leurs identités collectives ou individuelles, de leurs personnalités et de leurs caractéristiques. Je ne pense pas qu'ici le lien avec le libéralisme économique soit aussi évident. Aussi, je préfèrerais parler d'individualisme que de libéralisme.

\section{Peut-on mener des analyses globales?}

Peut-on mener des analyses globales puisqu'il y a des convergences fortes entre les pays? On n'a pas aujourd'hui de véritable réponse à cette question. Partant des débats, il apparaît évident qu'il y a de fortes convergences rhétoriques : il est clair que les rhétoriques officielles sont très proches entre les pays, notamment parce qu'elles s'inspirent de celle des agences internationales comme la Banque mondiale. Mais la vraie question, du point de vue du sociologue, de l'anthropologue ou du chercheur, se situe au niveau du terrain. Y a-t-il véritablement convergence? Sur le terrain, on voit que les politiques s'adaptent à des conditions particulières, aux sociétés et donc à des cultures nationales et 
même locales. Donc, comme disent les anthropologues, elles se créolisent, elles s'hybrident et finalement, même si le point de départ peut être le même, on peut aboutir à des résultats très différents. Ce n'est pas un paradoxe : il n'est qu'apparent de dire qu'il peut y avoir à la fois globalisation et diversification croissante des systèmes.

\section{Comment se réalise le lien entre la société et les politiques?}

On a entrevu sur ce sujet des différences importantes entre les systèmes. Dans l'atelier, on a pu opposer les systèmes des pays nordiques (Norvège, Suède et Pays-Bas), dans lesquels existe une recherche forte de consensus social portée par des collectifs structurés où le postulat de base est que le collectif pense mieux que l'individu. Au contraire, dans d'autres pays, l'État s'est fait l'interprète de la volonté générale soit de façon autoritariste soit, comme dans le modèle français, en incarnant simultanément la rationalité et la justice. Le deuxième modèle, le plus proche du cas français, a été fortement critiqué depuis les années soixante, notamment par les sociologues : «l'État est contrôlé par les classes dominantes» ou alors «l'État ne travaille qu'à sa propre reproduction». Est apparue ainsi, dans beaucoup de pays, la nécessité de développer un système beaucoup plus ouvert de consultations, de commissions et de groupes de travail, et donc un élargissement du cercle des personnes qui contribuent aux décisions. Ces dispositifs n'ont pas du tout les mêmes effets selon les types de société : celles où il existe une culture de la participation, celles qui sont structurées dans des collectifs, celles encore, comme dans les régimes post-communistes en transition, où les collectifs ont été déstructurés.

En conclusion, on ne peut pas imaginer bâtir les mêmes systèmes même si les processus semblent identiques.

\section{Quelle médiation entre les politiques et l'action?}

Entre la société et les politiques, il y a des médiateurs qui sont souvent des experts et en général des chercheurs. La recherche joue donc un rôle de médiation entre les politiques et l'action : cela pose problème du côté des politiques qui ont tendance à utiliser cette médiation comme une forme de légitimation, et du côté des enseignants dont la familiarité avec la recherche est insuffisante en raison de leur formation initiale et des caractéristiques de leur métier. Plus largement, les bases que fournit la recherche en éducation ne sont sans doute pas toujours suffisamment solides pour armer véritablement les politiques et les acteurs.

\section{Les enseignants}

Les enseignants sont-ils simplement spectateurs des réformes ou sontils des acteurs, participent-ils aux réformes, et plus généralement aux évolutions? S'il est évident que les enseignants sont pleinement acteurs des réformes, la question est de savoir à quel niveau et de quelle façon. 


\section{De quelle façon participent-ils aux changements?}

L'histoire de l'éducation nous montre que la force de résistance des enseignants est grande. Beaucoup de travaux de recherche concluent que «les réformes se sont arrêtées aux portes de la classe». Quand elles ne se sont pas arrêtées, elles se sont souvent transformées en autre chose, produisant des effets inattendus et souvent contraires à ceux souhaités par leurs initiateurs.

Si la force de résistance des enseignants est importante, on peut aussi observer une force d'accompagnement des politiques. Par exemple, de nombreux historiens ont décrit et analysé, pour le système américain, l'existence d'un one best system; on peut d'ailleurs se demander si on n'assiste pas à une convergence internationale, au moins au plan rhétorique, vers un one best system international. Cela n'est possible que par l'action quotidienne des enseignants dans la classe. On assiste à une sorte de miracle en voyant comment ces acteurs, qui agissent de façon très individualiste chacun dans leur classe, arrivent à produire des politiques aussi homogènes dans leurs effets. On peut faire le même constat que les systèmes aient des normes, comme par exemple les programmes en France, les examens aux États-Unis ou en Angleterre..., ou qu'ils n'en aient pas.

Indépendamment de ces normes, les instituts de formation des enseignants, en particulier les écoles normales d'instituteurs, ont joué un rôle de moule idéologique très fort auquel on pouvait se référer implicitement et, si nécessaire, explicitement. Les enseignants s'y conformaient d'eux-mêmes sans qu'il soit nécessaire de le leur rappeler.

Les transformations actuelles, qui sont particulièrement visibles en Angleterre par exemple, mais aussi dans beaucoup d'autres pays, vont dans le sens d'une rupture de ce contrat moral entre l'État et les enseignants. Le contrat était différent selon les pays mais il y avait effectivement une forte adhésion morale et politique des enseignants au projet de création et d'extension d'un système d'enseignement. Cette situation s'accompagnait d'une assez grande liberté dans le choix des moyens d'action : soit que celle-ci ait été officialisée comme en Angleterre où il y avait le modèle de l'enseignant professionnel, soit qu'elle ait été une liberté en creux comme en France où elle n'est pas officielle mais où, en fait, on tolère beaucoup de diversité dans l'action des enseignants. D'après les intervenants, ce contrat est rompu de deux façons : d'un côté il n'y a pas de discours très clair sur les valeurs, ce que les enseignants ne cessent de regretter. D'autre part, ce lien privilégié avec l'État est en train de disparaître alors qu'il protégeait l'enseignant de la société et notamment de la pression des usagers.

À sa place, dans beaucoup de pays, on a créé des systèmes de normes, sous le nom de «bonnes pratiques». L'enseignant se sent donc démuni au niveau des valeurs et fragilisé parce qu'il n'est plus protégé des pressions extérieures et également extrêmement contraint dans son activité quotidienne par rapport à la liberté dont il disposait auparavant. L'autonomie est dès lors ressentie par la 
très grande majorité des acteurs comme un renforcement des contraintes. C'est une autonomie au sein de laquelle on délègue aux enseignants la création des valeurs qu'on ne crée plus au niveau central, où on leur demande de décider ce qu'est l'intégration dans leur classe, où l'on reporte sur les chefs d'établissement la responsabilité de décider où s'arrête la laïcité, etc. Tous les acteurs sont donc placés dans cette position de très forte responsabilité morale, livrés à eux-mêmes. En même temps, cette autonomie est couplée avec une évaluation renforcée qui augmente l'impression de contrainte sur la capacité d'action. À la limite, les acteurs sentent qu'ils ont perdu sur tous les plans. De là découle le sentiment d'être incompris, mal aimés et oubliés, mais aussi les discours teintés de nostalgie pour un passé idéalisé porteurs de récriminations à l'égard des élèves et des parents.

La solution pourrait alors peut-être venir de la mise en place d'une instance de régulation collective : l'enseignant est peut-être maintenant devenu trop fragile au niveau individuel. C'est pour cette raison que des politiques et des chercheurs travaillent sur le développement d'une culture de collaboration et, par exemple dans les établissements, sur la création des formes de régulation collective. Les résistances sont grandes parce que beaucoup d'enseignants ne voient pas encore ce qu'ils auraient à y gagner : d'une part, ils pensent que ce serait une perte de leur autonomie individuelle à laquelle ils tiennent et, d'autre part, la principale caractéristique du métier - le fait qu'il s'exerce en solitaire semble incompatible avec de telles procédures. Beaucoup de jeunes enseignants ont encore aujourd'hui une vision charismatique du métier, affirmant par exemple : "tout ce qui concerne la discipline, ça ne peut pas se transmettre», "on a de l'autorité ou ne l'a pas, mais ça ne s'apprend pas", "on a choisi l'enseignement parce qu'on aime le contact avec les enfants et les jeunes plus qu'avec les adultes». Par leur référence individuelle, ces attitudes limitent les possibilités de développement d'une régulation collégiale.

\section{Participer aux politiques éducatives? Comment?}

Beaucoup d'enseignants ne souhaitent pas s'impliquer dans les politiques éducatives. Ils se méfient des politiques, craignent que la plupart des réformes ne viennent alourdir encore plus leur travail et augmenter leur responsabilité. Ils ne croient pas à des réformes globales : ils sont très sensibles à la diversité des contextes et convaincus qu'une réforme unique ne pourrait en tenir compte efficacement. Beaucoup adoptent l'attitude «ce n'est pas très bien mais tout ce qui viendra sera pire» et donc c'est une position de résistance profonde qui se développe par rapport à la participation.

\section{Avec quelle légitimité ?}

Les participants à l'atelier sont tombés d'accord sur le principe suivant : c'est à la société de décider des finalités de l'éducation et les enseignants sont alors des citoyens comme les autres. Cette évidence pose problème : en effet, si 


\section{Trois points de vue}

les enseignants ne sont pas convaincus que les réformes envisagées sont fondées et qu'on peut les mettre en œuvre, ils ne les appliqueront pas, et donc ils ne sont plus tout à fait des citoyens comme les autres dans la prise de décision.

\section{Avec quelle compétence?}

On a évoqué le rôle intellectuel des enseignants et ses aspects problématiques. Mais aujourd'hui, pour participer à l'élaboration des politiques, il faut également des capacités de négociation, de communication, de pilotage, etc. Or il n'est pas sûr non seulement que les enseignants disposent de ces compétences mais qu'ils souhaitent les avoir. Il faut savoir qu'interrogés, les enseignants adoptent des positions assez opposées à celles des cadres qui voient ce qu'ils peuvent gagner à s'associer aux réformes. On a rappelé parfois que le modèle de référence pour les enseignants est plutôt celui des professions libérales. Je crois qu'il faut compter avec cela. Les enseignants sont certes des acteurs fondamentaux des politiques, mais ils ne le sont pas toujours dans le sens où le voudraient les politiques. 


\section{Alan Wagner}

\section{L’évaluation}

Pourquoi évaluer? Qu'est-on censé évaluer? La généralisation de l'évaluation contribue t-elle à améliorer la qualité de l'enseignement? Des différentes logiques qui sous-tendent aujourd'hui le besoin d'évaluation, deux aspects majeurs ressortent : la pression sociale pour obtenir un haut niveau d'études et l'autonomie grandissante des établissements.

Dans la jeune économie de la connaissance, on accorde effectivement une grande importance au niveau de connaissances et de compétences; celui-ci conditionne l'obtention d'un emploi et l'accomplissement d'une carrière honorable. Plus largement, il est au cœur d'une vie d'adulte satisfaisante et active, dans un monde plus complexe et interdépendant.

Il convient ici d'établir une distinction majeure entre les résultats scolaires - dans une acception qui se limite à la maîtrise de connaissances liées aux disciplines - et un éventail plus large de connaissances et de compétences acquises, auquel s'ajoute la capacité à les mobiliser dans la vie quotidienne. Cette seconde famille réunit des compétences générales (capacité à résoudre des problèmes, aptitudes à communiquer, à travailler en équipe, compétences en technologies de l'information et de la communication et faculté d'apprendre à apprendre) et des compétences plus spécifiques à l'exercice d'un emploi. Cette distinction est importante car les répercussions sur l'évaluation individuelle des élèves, l'évaluation des établissements et l'évaluation du système scolaire ne sont pas les mêmes. L'acquisition de connaissances et de compétences, dans leur acception la plus large, se fait à la fois à l'intérieur de la classe et en dehors de l'école. Par ailleurs, l'évaluation de ces acquis dépasse l'évaluation du système éducatif en tant que tel (pensons par exemple au Programme international pour le suivi des acquis des élèves (PISA), de l'OCDE). Les établissements et les systèmes éducatifs doivent organiser en conséquence l'enseignement de manière à prendre en compte, soutenir et encourager l'acquisition d'un éventail plus large de connaissances et de compétences.

La nécessité d'évaluer s'explique aussi par l'autonomie croissante dont jouissent les établissements scolaires. En même temps qu'elle leur donne des moyens, cette autonomie a pour objectif d'inciter toutes les personnes concernées - au sein de l'établissement comme dans son environnement immédiat - à coordonner le mieux possible les programmes et à structurer l'enseignement et l'apprentissage de façon à améliorer les résultats des élèves. Il s'agit bel et bien de responsabiliser les établissements par rapport aux performances scolaires. Si l'autonomie des établissements vise à encourager de tels progrès, les évaluations sont nécessaires pour éclairer les décisions, identifier les faiblesses et les besoins 


\section{Trois points de vue}

et faire connaître les succès et les échecs de certaines approches ou initiatives. Qu'elles soient formatives (pour inciter au progrès) ou sommatives (pour vérifier si les objectifs ont été atteints), les évaluations participent à la prise de décision aux niveaux des établissements et du système éducatif.

\section{Qu'est-on censé évaluer?}

Cette question, qui a été soulevée pour les établissements, pourrait s'appliquer aux différents acteurs au niveau de l'établissement et au-delà. Une information objective est aujourd'hui nécessaire pour mesurer ce qu'un jeune connaît et sait faire. Comme nous l'avons déjà suggéré, cette évaluation dépasse la connaissance des disciplines et renvoie à un apprentissage qui se déroule en dehors du cadre scolaire. Mesurer la participation des élèves pendant les cours, identifier le seuil de passage à la classe supérieure et le niveau atteint à la fin de la scolarité ne suffisent plus.

Aujourd'hui, les évaluations sont moins susceptibles de porter sur l'étendue des seules connaissances et des compétences. En outre, les différents acteurs ne partagent pas la même vision de ce que les établissements et les systèmes éducatifs sont censés faire. De ce point de vue, ce qui n'est pas évalué - ce qui ne fait pas l'objet de tests - est aussi révélateur que ce qui l'est. Dans certains pays l'évaluation ne couvre pas les compétences générales ni ce qui relève de la culture générale (dont l'instruction civique), ni en outre ce qui se rapporte au premier emploi et à l'expérience professionnelle.

\section{Pour qui et comment évalue-t-on?}

Les évaluations qui portent sur les résultats de l'apprentissage - c'est à dire les connaissances et les compétences acquises - fournissent les bases pour évaluer à la fois le système éducatif, les établissements, les équipes d'enseignants, ainsi que chaque enseignant et chaque élève. Elles doivent permettre, notamment à l'élève - et à ses parents - de savoir où il en est, de comprendre ce qu'il a appris et ce qu'il n'a pas encore assimilé.

Qui a l'expertise pour élaborer, mettre en œuvre et interpréter ces évaluations? Cette expertise se trouve à l'intérieur même du système éducatif; elle est entre les mains des enseignants. Ils sont formés pour organiser l'apprentissage et l'évaluer, et ont l'expérience nécessaire. Ils savent comment des connaissances éparses et, plus largement, des familles de compétences se combinent pour former un ensemble cohérent qui sert à la poursuite des études ou de l'apprentissage, à l'exercice d'un métier et à la vie d'adulte.

Dans le système scolaire, l'évaluation repose sur des indicateurs de performance qui peuvent être comparés à des normes à l'échelle des établissements, des académies et des pays. L'évaluation au sein des systèmes éducatifs continue de se développer avec l'introduction de nouveaux tests et/ou de nouvelles méthodes, comme en Catalogne, au Sénégal, au Maroc, en Allemagne, 
au Royaume-Uni et aux États-Unis. Cette tendance répond à la fois à de nouveaux besoins d'informations, à de nouvelles défaillances du système scolaire, à des insuffisances récemment mises à jour, ou encore à des faiblesses de l'évaluation formative actuelle. Une question importante se pose, celle de la circulation et de l'interprétation des informations au niveau des systèmes éducatifs. Il existe en effet une perspective de recherche très prometteuse sur la valeur ajoutée, qui prendrait pour point de départ l'établissement mais irait au-delà, en tenant compte de toutes les structures d'apprentissage et des acquis de chaque élève. L'objectif est que les établissements puissent vraiment exploiter ces données et que leurs résultats soient reconnus. Lorsque l'environnement est difficile, ces informations et ces évaluations permettent de valoriser les aspects positifs du contexte et du bagage éducatif des élèves. Des réalisations de ce type existent dans plusieurs pays, aux États-Unis et dans la communauté francophone de Belgique notamment, et dans une certaine mesure au niveau mondial, à travers les évaluations conduites par l'Institute of International Association for the Evaluation of Educational Achievement (IEA), et par l'OCDE avec PISA. Des études sont menées sur les attentes que formulent les élèves, les parents, les entreprises et la collectivité en général ainsi que sur leurs perceptions des performances des établissements, des enseignants et du système éducatif. C'est le cas au Royaume-Uni et cette perspective est à l'étude en France. Il n'est évidemment pas question de substituer ces travaux aux évaluations individuelles, mais les établissements et les instances supérieures du système éducatif s'en servent comme outils d'aide à la décision.

Dans la mesure où les résultats par établissement facilitent une lecture comparée, l'évaluation se traduit également à travers le choix des élèves et des étudiants - et de leurs parents - de s'inscrire dans tel établissement plutôt que dans tel autre. C'est ce qu'on appelle aux États-Unis «voter avec ses pieds». Certains parents décident de l'endroit où ils souhaitent vivre en fonction de leur appréciation de l'enseignement local. Ce sont les agents immobiliers qui fournissent ce type d'informations aux futurs propriétaires!

Dans les établissements, dans une perspective d'amélioration des résultats des élèves, le développement professionnel des enseignants dépend de leur évaluation (à travers l'auto-évaluation, l'inspection, les tests et l'évaluation par les élèves). S’il s'avère cependant difficile de démontrer la très forte corrélation qui existe entre le développement professionnel de l'enseignant d'une part et les résultats de l'élève, de l'établissement ou encore du système d'autre part, l'évaluation du savoir faire et de l'expérience de l'enseignant, ajoutée à des objectifs précis de développement professionnel - ciblant l'élève, le groupe ou l'établissement - donne aujourd'hui les meilleures chances de résultats en termes d'amélioration du niveau de connaissances des élèves.

En outre, les établissements et les enseignants doivent répondre à de nouveaux besoins et de nouvelles attentes, nécessitant pour la plupart l'acquisition de compétences autres que pédagogiques et un savoir qui dépasse la connaissance 
d'une discipline. Il s'agit de s'adapter à l'hétérogénéité des élèves, d'apporter un soutien individualisé, de prendre en compte l'apprentissage informel qui se fait en dehors de l'école (en ayant recours à l'informatique notamment), de travailler avec les parents etc. Dans une certaine mesure, ces besoins et ces attentes ont toujours été implicites. Ils sont devenus explicites le jour où l'on a précisé ce qu'un élève est censé connaître et savoir faire, c'est à dire à la fois l'étendue de ses connaissances et leur degré d'approfondissement. Telles sont certaines des compétences nouvelles demandées aux enseignants et pour lesquelles ils ont à être évalués.

Dans quelle mesure l'évaluation de l'enseignement et de l'apprentissage dans les établissements doit-il porter sur l'enseignant ou sur l'équipe pédagogique - en d'autres termes, doit-on privilégier l'individuel ou le collectif? Les deux sont utiles.

\section{Comment concilier logiques économiques et logiques pédagogiques?}

Quel est le lien entre financement et apprentissage? La concurrence en vue d'obtenir les ressources nécessaires est de plus en plus rude, à tel point que les fonds publics consacrés à l'éducation risquent, dans le meilleur des cas, de se maintenir à leur niveau actuel. Le recours croissant aux financements privés semble inévitable, soit en finançant les établissements ou leurs activités via les frais de scolarité, soit en utilisant des fonds privés pour des activités spécifiques et l'apprentissage «informel» en dehors de l'école.

Il n'est pas évident de comprendre comment les niveaux et les sources de financement ont un impact sur les résultats globaux des systèmes éducatifs, ni comment ils expliquent les différences de performances entre établissements. Les pays qui atteignent des niveaux de performances scolaires relativement élevés correspondent à ceux dont le montant des ressources se situe dans la moyenne, ressources qui sont presque exclusivement publiques - comme en Finlande - ou largement privées - comme en Corée. Aux États-Unis et au Brésil, des travaux de recherche montreraient que certains types d'investissement (tels le temps que les élèves consacrent aux activités d'apprentissage ou le savoir et les compétences des enseignants) contribuent réellement à améliorer les résultats. Dans un nombre croissant de pays, les politiques menées au niveau du système éducatif cherchent à lier le financement et la répartition des ressources aux résultats des établissements bénéficiant d'une certaine autonomie. Cette corrélation constitue une incitation supplémentaire pour les acteurs de ces établissements, déjà confrontés aux besoins et aux attentes externes des élèves, parents et employeurs.

Le défi à relever est d'améliorer les faibles résultats scolaires, alors que le niveau exigé des performances monte, que les paramètres à évaluer se font plus nombreux et que l'écart de performances entre établissements peut être 
aussi grand à l'intérieur d'une académie qu'entre académies. Doit-on envisager des stratégies géographiques regroupant plusieurs établissements (telles les zones d'éducation prioritaires - ZEP en France), notamment ne ce qui concerne les établissements à faible niveau de performance? Ou encore, est-ce que des approches individualisées correspondant à des besoins d'apprentissages individualisés permettraient aux élèves et aux établissements d'obtenir de meilleurs résultats? Cela se fait dans les écoles américaines à travers les programmes individuels d'éducation, conçus pour chaque élève ayant des besoins particuliers. Selon la stratégie adoptée, les répercussions sur la répartition des ressources ne sont pas les mêmes, ni entre établissements ni au sein des établissements.

\section{Comment favoriser une répartition efficace des ressources?}

Dans un certain nombre de pays où la répartition des ressources obéit à plusieurs critères - le nombre d'élèves, leurs caractéristiques, la nature et le niveau des programmes - une nouvelle approche émerge, qui recherche l'efficacité maximale des ressources. Cela se traduit, dans de nombreux pays, par le recours explicite aux évaluations et à une politique budgétaire très encadrée, avec des régulations et des contrôles. Grâce à leur plus grande autonomie, les acteurs au sein des établissements sont en mesure de prendre les décisions visant à améliorer leurs résultats, et toutes choses égales par ailleurs, à accroître leur efficience.

L'autonomie des établissements risque d'être insuffisante, à elle seule, pour favoriser une répartition plus efficace des ressources. Il reste nécessaire de contrôler les résultats des établissements et d'articuler les ressources avec les stratégies conçues et mises en œuvre dans le but d'améliorer les résultats des établissements. La corrélation entre ressources et résultats constitue une véritable réorientation, qui nécessite en effet une régulation constante.

Nous avons évoqué la nécessité de développer les compétences en faveur de la prise de décision au niveau de l'établissement et, je dirais même, dans la classe. Faciliter le développement professionnel des directeurs d'établissement et des enseignants est à l'ordre du jour. L'ancien président de l'université du Michigan a déclaré qu'à l'avenir, les universités les plus dynamiques compteront des enseignants profondément conscients de la dimension financière de l'éducation. Les enseignants jouent un rôle crucial. Comment faire en sorte qu'ils soient intégrés au processus de décision, qu'ils mobilisent vraiment leurs compétences et se sentent encouragés? Cela implique de repenser la professionnalité enseignante.

En conclusion, la logique financière ne remplace pas les autres logiques au service de l'organisation du travail des établissements et n'offre pas non plus un cadre suffisant pour l'évaluation des résultats des établissements, pas plus qu'elle ne détermine les rôles et les missions des établissements. 